PROCEEDINGS OF THE

AMERICAN MATHEMATICAL SOCIETY

Volume 139, Number 12, December 2011, Pages 4343-4349

S 0002-9939(2011)10840-2

Article electronically published on April 4, 2011

\title{
NONZERO POSITIVE SOLUTIONS OF SYSTEMS OF ELLIPTIC BOUNDARY VALUE PROBLEMS
}

\author{
K. Q. LAN
}

(Communicated by Yingfei Yi)

\begin{abstract}
A new result on existence of nonzero positive solutions of systems of second order elliptic boundary value problems is obtained under some sublinear conditions involving the principle eigenvalues of the corresponding linear systems. Results on eigenvalue problems of such elliptic systems are derived and generalize some previous results on the eigenvalue problems of systems of Laplacian elliptic equations. Applications of our results are given to two such systems with specific nonlinearities.
\end{abstract}

\section{INTRODUCTION}

We consider existence of nonzero positive solutions of systems of second order elliptic equations

$$
\mathbb{L} z_{i}(x)=f_{i}(x, \mathbf{z}(x)) \quad \text { on } \bar{\Omega}, i \in I_{n}:=\{1, \cdots, n\}
$$

subject to boundary conditions involving first order boundary operators, where $\mathbb{L}$ is a strongly uniformly elliptic differential operator and $\Omega$ is a suitable bounded open set in $\mathbb{R}^{m}$. We seek solutions of (1.1) in $C\left(\bar{\Omega} ; \mathbb{R}_{+}^{n}\right) \backslash\{0\}$.

When $n=1$ and $f_{1}$ satisfies suitable monotonicity conditions, (1.1) was studied, for example by Amann in [1, 2].

A special case of (1.1) with the Dirichlet boundary condition is the system of semilinear elliptic equations of the form

$$
\left\{\begin{array}{l}
-\triangle z_{i}(x)=\lambda f_{i}(\mathbf{z}(x)) \quad \text { on } \bar{\Omega}, i \in I_{n}, \\
z_{i}(x)=0 \text { on } \partial \Omega .
\end{array}\right.
$$

An open question proposed by Lions in [8] is whether (1.2) with $\lambda=1$ has a nonzero positive solution under sublinear or superlinear conditions which involve the principal eigenvalues of the corresponding linear systems (see [8, question $(c)$ in section 4.2]).

There have been some results on the above question under the sublinear cases. Hai and Wang 7 prove that $(1.2)$ has a nonzero positive solution in $C\left(\bar{\Omega} ; \mathbb{R}^{n}\right)$ for each $\lambda \in(0, \infty)$ under the following sublinear condition:

Received by the editors June 30, 2010 and, in revised form, October 9, 2010.

2010 Mathematics Subject Classification. Primary 35J57; Secondary 45G15, $47 \mathrm{H} 10$.

Key words and phrases. Systems of elliptic boundary value problems, sublinear condition, nonzero positive solutions, fixed point index.

The author was supported in part by the Natural Sciences and Engineering Research Council (NSERC) of Canada.

(C)2011 American Mathematical Society Reverts to public domain 28 years from publication 4343 
(i) $\lim _{|\mathbf{z}|_{1} \rightarrow 0} \frac{f_{i_{0}}(\mathbf{z})}{|\mathbf{z}|_{1}}=\infty$ for some $i_{0} \in I_{n}$ and $f_{i_{0}}(\mathbf{z})>0$ for $\mathbf{z} \in \mathbb{R}_{+}^{n}$ and

(ii) $\lim _{|\mathbf{z}|_{1} \rightarrow \infty} \frac{f_{i}(\mathbf{z})}{|\mathbf{z}|_{1}}=0$ for each $i \in I_{n}$,

where $p$-Laplacian systems are considered and $|\mathbf{z}|_{1}=\sum_{i=1}^{n}\left|z_{i}\right|$ (see 7, Theorem 1.2]). We refer to [4, 5, 6, 10, for the existence and uniqueness of elliptic systems related to (1.2) under other sublinear conditions.

In this paper, using the theory of fixed point index for compact maps defined on cones in Banach spaces 1, we prove that (1.1) has a nonzero positive solution in $C\left(\bar{\Omega} ; \mathbb{R}^{n}\right)$ under a sublinear condition which contains, as a special case, the following conditions:

$(i)^{\prime} \lim _{|\mathbf{z}| \rightarrow 0} \frac{f_{i_{0}}(\mathbf{z})}{|\mathbf{z}|}>\mu_{1}$ for some $i_{0} \in I_{n}$ and

$(\text { ii })^{\prime} \lim _{|\mathbf{z}| \rightarrow \infty} \frac{f_{i}(\mathbf{z})}{|\mathbf{z}|}<\mu_{1}$ for each $i \in I_{n}$,

where $\mu_{1}$ is the largest characteristic value of the linear system corresponding to (1.1) and $|\mathbf{z}|=\max \left\{\left|z_{i}\right|: i \in I_{n}\right\}$. Hence, our result improves the results in 7]. As illustrations, we apply our result to (1.1) with some specific nonlinearities.

\section{NonZERo POSITIVE SOLUTIONS OF SYSTEMS OF SECOND ORDER ELLIPTIC BOUNDARY VALUE PROBLEMS}

We study existence of nonzero positive solutions of systems of second order elliptic boundary value problems of the form

$$
\mathbb{L} z_{i}(x)=f_{i}(x, \mathbf{z}(x)) \text { on } \bar{\Omega}, i \in I_{n}
$$

subject to the following boundary condition:

$$
B z_{i}(x)=0 \text { on } \partial \Omega,
$$

where $\mathbf{z}(x)=\left(z_{1}(x), \cdots, z_{n}(x)\right), \mathbb{L}$ is a strongly uniformly elliptic differential operator, $B$ is a first order boundary operator and $f_{i}: \bar{\Omega} \times \mathbb{R}_{+}^{n} \rightarrow \mathbb{R}_{+}$is continuous. If $m=1$, let $\Omega=\left(x_{0}, x_{1}\right)$, where $x_{0}, x_{1} \in \mathbb{R}$ with $x_{0}<x_{1}$. If $m \geq 2$, we assume that $\Omega$ is a bounded open set in $\mathbb{R}^{m}$ and the boundary $\partial \Omega$ of $\Omega$ is assumed to be an $(m-1)$-dimensional $C^{2+\mu}$-manifold for some $\mu \in(0,1)$ such that $\Omega$ lies locally on one side of $\partial \Omega$ (see [1, section 4 of Chapter 1]).

Let $\hat{\mu}=0$ if $m=1$ and $\hat{\mu}=\mu$ if $m \geq 2$. Recall that a second order elliptic differential operator $\mathbb{L}$ defined by

$$
\mathbb{L} u=-\sum_{k, j=1}^{m} a_{k j}(x) \frac{\partial^{2} u}{\partial x_{k} \partial x_{j}}+\sum_{k=1}^{m} b_{k}(x) \frac{\partial u}{\partial x_{k}}+c(x) u
$$

is said to be strongly uniformly elliptic if $a_{k j}, b_{k}, c \in C^{\hat{\mu}}(\bar{\Omega})$ for $k, j \in I_{m}, c(x) \geq 0$ for $x \in \bar{\Omega}, a_{k j}(x)=a_{j k}(x)$ for $x \in \bar{\Omega}$ and $k, j \in I_{m}$, and there exists $\mu_{0}>0$ such that

$$
\sum_{k, j=1}^{m} a_{k j}(x) \xi_{k} \xi_{j} \geq \mu_{0}|\xi|^{2} \quad \text { for } x \in \bar{\Omega} \text { and } \xi=\left(\xi_{1}, \ldots, \xi_{m}\right) \in \mathbb{R}^{m} .
$$

If $m=1$, the first order boundary operator $B$ is

$$
B u(x)= \begin{cases}\alpha_{0} u\left(x_{0}\right)-\beta_{0} u^{\prime}\left(x_{0}\right) & \text { if } x=x_{0}, \\ \alpha_{1} u\left(x_{1}\right)-\beta_{1} u^{\prime}\left(x_{1}\right) & \text { if } x=x_{1},\end{cases}
$$


where $\alpha_{0}, \alpha_{1}, \beta_{0}, \beta_{1} \in[0, \infty)$ satisfy $\left(\alpha_{0}+\beta_{0}\right)\left(\alpha_{1}+\beta_{1}\right)>0$. If $m \geq 2$, then

$$
B u=b u+\delta \frac{\partial u}{\partial v},
$$

where $v$ is an outward pointing, nowhere tangent vector field on $\partial \Omega$ of $C^{1+\mu}, \partial u / \partial v$ denotes the directional derivative of $u$ with respective to $v$, and $\delta$ and $b$ satisfy one of the following conditions: $(i) \delta=0$ and $b \equiv 1$ (Dirichlet boundary operator); (ii) $\delta=1, b \equiv 0$ and $c \neq \equiv 0$ on $\bar{\Omega}$ (Neumann boundary operator); or (iii) $\delta=1$, $b \in C^{1+\mu}(\partial \Omega), b(x) \geq 0$ and $b(x) \not \equiv 0$ on $\partial \Omega$ (regular oblique derivative boundary operator).

Lemma 2.1 ([1]). For every $v \in C^{\hat{\mu}}(\bar{\Omega})$, the linear boundary value problem

$$
\left\{\begin{array}{l}
\mathbb{L} u(x)=v(x) \quad \text { on } \bar{\Omega}, \\
B u(x)=0 \quad \text { on } \partial \Omega
\end{array}\right.
$$

has a unique solution $u \in C^{2+\hat{\mu}}(\bar{\Omega})$.

For every $v \in C^{\hat{\mu}}(\bar{\Omega})$, we denote by $T^{*} v$ the unique solution of (2.6). It is known that $T^{*}: C^{\hat{\mu}}(\bar{\Omega}) \rightarrow C^{2+\hat{\mu}}(\bar{\Omega})$ is a bounded and surjective linear operator and has a unique extension, denoted by $T$, to $C(\bar{\Omega})$. We write

$$
e=T^{*} v_{0}, \quad \text { where } v_{0}(x) \equiv 1 .
$$

It is known that $e$ is an interior point of the positive cone $P_{1}$ in $C(\bar{\Omega})$, where

$$
P_{1}=\{z \in C(\bar{\Omega}): z(x) \geq 0 \quad \text { for } x \in \bar{\Omega}\} .
$$

The following result gives the properties of $T$ which are contained in [1, Theorem 4.2] and [2, Lemma 5.3].

Lemma 2.2. $T: C(\bar{\Omega}) \rightarrow C^{1}(\bar{\Omega}) \subset C(\bar{\Omega})$ is a compact linear operator such that $T\left(P_{1}\right) \subset P_{1}$ and for each $v \in P_{1} \backslash\{0\}$, there exists $\alpha_{v}>0$ such that $T v \geq \alpha_{v} e$.

By Lemma 2.2 and the well-known Krein-Rutman theorem (see [1, Theorem 3.1] or [9]), it is easy to see that $\mu_{1} \in(0, \infty)$ and there exists $\varphi_{1} \in P_{1} \backslash\{0\}$ such that

$$
\varphi_{1}=\mu_{1} T \varphi_{1}
$$

where $\mu_{1}=1 / r(T)$ and $r(T)$ is the spectral radius of $T$.

We use the following maximum norm in $\mathbb{R}^{n}$ :

$$
|\mathbf{z}|=\max \left\{\left|z_{i}\right|: i \in I_{n}\right\},
$$

where $\mathbf{z}=\left(z_{1}, \cdots, z_{n}\right)$. We denote by $C\left(\bar{\Omega} ; \mathbb{R}^{n}\right)$ the Banach space of continuous functions from $\bar{\Omega}$ into $\mathbb{R}^{n}$ with norm $\|\mathbf{z}\|=\max \left\{\left\|z_{i}\right\|: i \in I_{n}\right\}$, where

$$
\mathbf{z}(x)=\left(z_{1}(x), \cdots, z_{n}(x)\right) \quad \text { for } x \in \bar{\Omega} .
$$

We use the standard positive cone in $C\left(\bar{\Omega} ; \mathbb{R}^{n}\right)$ defined by

$$
P=C\left(\bar{\Omega} ; \mathbb{R}_{+}^{n}\right) .
$$

We define $L: C\left(\bar{\Omega}, \mathbb{R}^{n}\right) \rightarrow C\left(\bar{\Omega}, \mathbb{R}^{n}\right)$ by

$$
(L \mathbf{z})(x):=\left(\left(T z_{1}\right)(x), \cdots,\left(T z_{n}\right)(x)\right)
$$

and a Nemytskii operator $F: P \rightarrow P$ by

$$
(F \mathbf{z})(x)=\left(f_{1}(x, \mathbf{z}(x)), \cdots, f_{n}(x, \mathbf{z}(x))\right) .
$$


It is easy to verify that (2.1) is equivalent to the following fixed point equation:

$$
\mathbf{z}(x)=(L F \mathbf{z})(x):=A \mathbf{z}(x) \text { for } x \in \bar{\Omega} .
$$

Recall that a solution $\mathbf{z} \in C\left(\bar{\Omega} ; \mathbb{R}^{n}\right)$ of (2.1) is said to be a nonzero positive solution if $\mathbf{z} \in P \backslash\{0\}$; that is, $\mathbf{z} \in C\left(\bar{\Omega} ; \mathbb{R}^{n}\right)$ and $\mathbf{z}(x)=\left(z_{1}(x), \cdots, z_{n}(x)\right)$ satisfies $z_{i}(x) \geq 0$ for $x \in \bar{\Omega}$ and $i \in I_{n}$ and there exists $k \in I_{n}$ such that $z_{k}(x) \not \equiv 0$ on $\bar{\Omega}$.

Let $\rho>0$ and let $P_{\rho}=\{x \in P:\|x\|<\rho\}, \bar{P}_{\rho}=\{x \in P:\|x\| \leq \rho\}$ and $\partial P_{\rho}=\{x \in P:\|x\|=\rho\}$.

We need some results from the theory of the fixed point index for compact maps defined on cones in a Banach space $X$ (see [1]).

Lemma 2.3. Assume that $A: \bar{P}_{\rho} \rightarrow P$ is a compact map. Then the following results hold:

(1) If there exists $x_{0} \in P \backslash\{0\}$ such that $z \neq A z+\nu x_{0}$ for $z \in \partial P_{\rho}$ and $\nu \geq 0$, then $i_{P}\left(A, P_{\rho}\right)=0$.

(2) If $z \neq \varrho A z$ for $x \in \partial P_{\rho}$ and $\varrho \in(0,1]$, then $i_{P}\left(A, P_{\rho}\right)=1$.

(3) If $i_{P}\left(A, P_{\rho}\right)=1$ and $i_{P}\left(A, P_{\rho_{0}}\right)=0$ for some $\rho_{0} \in(0, \rho)$, then $A$ has a fixed point in $P_{\rho} \backslash \bar{P}_{\rho_{0}}$.

Now, we are in a position to give our main result.

Theorem 2.1. Let $\mu_{1}$ be the same as in (2.8). Assume that the following conditions hold:

$\left(\left(f_{i_{0}}\right)_{0}\right)_{\rho_{0}}$ There exist $i_{0} \in I_{n}, \varepsilon>0$ and $\rho_{0}>0$ such that

$$
f_{i_{0}}(x, \boldsymbol{z}) \geq\left(\mu_{1}+\varepsilon\right) z_{i_{0}} \quad \text { for } x \in \bar{\Omega} \text { and all } \boldsymbol{z} \in \mathbb{R}_{+}^{n} \text { with }|\boldsymbol{z}| \in\left[0, \rho_{0}\right] .
$$

$\left(f_{i}^{\infty}\right)_{\rho_{1}}$ There exist $\varepsilon>0$ and $\rho_{1}>0$ such that for each $i \in I_{n}$,

$$
f_{i}(x, z) \leq\left(\mu_{1}-\varepsilon\right)|z| \quad \text { for } x \in \bar{\Omega} \text { and all } \boldsymbol{z} \in \mathbb{R}_{+}^{n} \text { with }|\boldsymbol{z}| \geq \rho_{1} .
$$

Then (2.1) has a nonzero positive solution in $P$.

Proof. By Lemma 2.2, $L: C\left(\bar{\Omega} ; \mathbb{R}^{n}\right) \rightarrow C\left(\bar{\Omega} ; \mathbb{R}^{n}\right)$ is compact and satisfies $L(P) \subset P$. This, together with the continuity of $f_{i}$, implies that $A: P \rightarrow P$ is compact. Without loss of generalization, we assume that $\mathbf{z} \neq A \mathbf{z}$ for $\mathbf{z} \in \partial P_{\rho_{0}}$. Let $\varphi=$ $\left(\varphi_{1}, \cdots, \varphi_{1}\right)$, where $\varphi_{1}$ is the same as in (2.8). We prove that

$$
\mathbf{z} \neq A \mathbf{z}+\nu \varphi \text { for all } \mathbf{z} \in \partial P_{\rho_{0}} \text { and } \nu \geq 0 .
$$

In fact, if not, there exist $\mathbf{z} \in \partial P_{\rho_{0}}$ and $\nu>0$ such that $\mathbf{z}=A \mathbf{z}+\nu \varphi$. Then

$$
z_{i_{0}}(x)=T\left(f_{i_{0}}(x, \mathbf{z}(x))\right)+\nu \varphi_{1}(x) \text { for all } x \in \bar{\Omega} .
$$

It follows that $z_{i_{0}}(x) \geq \nu \varphi_{1}(x)$ for $x \in \bar{\Omega}$. Let

$$
\tau=\sup \left\{\tau_{0}>0: z_{i_{0}}(x) \geq \tau_{0} \varphi_{1}(x) \text { for all } x \in \bar{\Omega}\right\} .
$$

Then $0<\nu \leq \tau<\infty$ and $z_{i_{0}}(x) \geq \tau \varphi_{1}(x)$ for all $x \in \bar{\Omega}$. This, together with (2.15), $\left(\left(f_{i_{0}}\right)_{0}\right)_{\rho_{0}}$ and (2.8), implies that for all $x \in \bar{\Omega}$,

$$
z_{i_{0}}(x) \geq T\left(\left(\mu_{1}+\varepsilon\right) z_{i_{0}}(x)\right) \geq\left(\mu_{1}+\varepsilon\right) \tau T \varphi_{1}(x)=\left(\mu_{1}+\varepsilon\right) \tau\left(\varphi_{1}(x) / \mu_{1}\right) .
$$

Hence, we have $\tau \geq\left(\mu_{1}+\varepsilon\right) \tau / \mu_{1}>\tau$, a contradiction. It follows from (2.14) and Lemma 2.3 (1) that $i_{P}\left(A, P_{\rho_{0}}\right)=0$.

For each $i \in I_{n}$, by the continuity of $f_{i}$, there exists $b_{i}>0$ such that

$$
f_{i}(x, \mathbf{z}) \leq b_{i} \quad \text { for } x \in \bar{\Omega} \text { and } \mathbf{z} \in \mathbb{R}_{+}^{n} \text { with }|\mathbf{z}| \leq \rho_{1} .
$$


This, together with $\left(f_{i}^{\infty}\right)_{\rho_{1}}$, implies that, for each $i \in I_{n}$,

$$
f_{i}(x, \mathbf{z}) \leq b_{i}+\left(\mu_{1}-\varepsilon\right)|\mathbf{z}| \quad \text { for } x \in \bar{\Omega} \text { and all } \mathbf{z} \in \mathbb{R}_{+}^{n} .
$$

Since $r\left(\left(\mu_{1}-\varepsilon\right) T\right)=\left(\mu_{1}-\varepsilon\right) r(T)<1,\left(I-\left(\mu_{1}-\varepsilon\right) T\right)^{-1}$ exists and is bounded and satisfies $\left(I-\left(\mu_{1}-\varepsilon\right) T\right)^{-1} P_{1} \subset P_{1}$. Let $\hat{b}_{i}(x) \equiv b_{i}$ for $x \in \bar{\Omega}, \rho_{1}=\max \left\{\left\|T \hat{b}_{i}\right\|\right.$ : $\left.i \in I_{n}\right\}$ and $\rho^{*}=\left\|\left(I-\left(\mu_{1}-\varepsilon\right) T\right)^{-1} \hat{\rho_{1}}\right\|$, where $\hat{\rho_{1}}(x) \equiv \rho_{1}$ for $x \in \bar{\Omega}$. Let $\rho>\rho^{*}$. We prove

$$
\mathbf{z} \neq \varrho A \mathbf{z} \quad \text { for } \mathbf{z} \in \partial P_{\rho} \text { and } \varrho \in(0,1] .
$$

Indeed, if not, there exist $\mathbf{z} \in \partial P_{\rho}$ and $\varrho \in(0,1]$ such that $\mathbf{z}=\varrho A \mathbf{z}$. By (2.16), we have for each $i \in I_{n}$,

$$
z_{i}(x) \leq \rho_{1}+\left(\mu_{1}-\varepsilon\right)(T|\mathbf{z}|)(x) \quad \text { for } x \in \bar{\Omega},
$$

where $|\mathbf{z}|(x)=\max \left\{\left|z_{i}(x)\right|: i \in I_{n}\right\}$. Taking the maximum in the above inequality implies that

$$
|\mathbf{z}|(x) \leq \rho_{1}+\left(\mu_{1}-\varepsilon\right)(T|\mathbf{z}|)(x) \text { for } x \in \bar{\Omega}
$$

and $\left(I-\left(\mu_{1}-\varepsilon\right) T\right)|\mathbf{z}|(x) \leq \rho_{1}$ for $x \in \bar{\Omega}$. Since $\left(I-\left(\mu_{1}-\varepsilon\right) T\right)^{-1} P_{1} \subset P_{1}$,

$$
|\mathbf{z}|(x) \leq\left(I-\left(\mu_{1}-\varepsilon\right) T\right)^{-1} \hat{\rho_{1}}(x) \text { for } x \in \bar{\Omega} .
$$

Hence, we have

$$
\rho=\|\mathbf{z}\|=\max \{|\mathbf{z}|(x): x \in \bar{\Omega}\} \leq \rho^{*}<\rho,
$$

a contradiction. By (2.17) and Lemma 2.3 $(2), i_{P}\left(A, P_{\rho}\right)=1$. By Lemma 2.3 (3), (2.1) has a solution in $P_{\rho} \backslash \bar{P}_{\rho_{0}}$.

Notation. Let

$$
\begin{aligned}
\underline{f_{i}}(\mathbf{z}) & =\inf _{x \in \bar{\Omega}} f_{i}(x, \mathbf{z}), \overline{f_{i}}(\mathbf{z})=\sup _{x \in \bar{\Omega}} f_{i}(x, \mathbf{z}) \\
\left(f_{i}\right)_{0} & =\liminf _{|\mathbf{z}| \rightarrow 0^{+}} \underline{f_{i}}(\mathbf{z}) /|\mathbf{z}|, \quad\left(f_{i}\right)^{\infty}=\limsup _{|\mathbf{z}| \rightarrow \infty} \overline{f_{i}}(\mathbf{z}) /|\mathbf{z}| .
\end{aligned}
$$

As a special case of Theorem 2.1 we obtain the following result.

Corollary 2.1. Assume that the following conditions hold:

(i) There exists $i_{0} \in I_{n}$ such that $\left(f_{i_{0}}\right)_{0}>\mu_{1}$.

(ii) $\left(f_{i}\right)^{\infty}<\mu_{1}$ for all $i \in I_{n}$.

Then (2.1) has a nonzero positive solution in $P$.

Corollary 2.1 with $n=1$ improves Theorem 1.3 in [8], where $f: \mathbb{R} \rightarrow \mathbb{R}$ is locally Lipschitz continuous satisfying $f(0)=0$, and Corollary II.1 in [3, where $f$ satisfies the Carathéodory conditions, but the positive solutions are in $W^{2, p}(\Omega)$ for every $1<p<\infty$. When $m=1, \mathbb{L} z=-z^{\prime \prime}$, Corollary 2.1 with $n=1$ and (2.4) improve Theorem $4.1\left(H_{2}\right)$ in [11, where $\left[x_{0}, x_{1}\right]=[0,1]$.

As an application of Corollary 2.1, we consider the following eigenvalue problem:

$$
\mathbb{L} z_{i}(x)=\lambda f_{i}(x, \mathbf{z}(x)) \text { on } \bar{\Omega}, i \in I_{n},
$$

subject to (2.2).

Corollary 2.2. Assume there exists $i_{0} \in I_{n}$ such that

$$
0 \leq \bar{f}^{\infty}<\left(f_{i_{0}}\right)_{0} \leq \infty,
$$

where $\bar{f}^{\infty}=\max \left\{\left(f_{i}\right)^{\infty}: i \in I_{n}\right\}$. Then for each $\lambda \in\left(\frac{\mu_{1}}{\left(f_{i_{0}}\right)_{0}}, \frac{\mu_{1}}{f^{\infty}}\right)$, (2.18) $-(2.2)$ has a nonzero positive solution in $P$. 
Proof. Since for each $\lambda \in\left(\frac{\mu_{1}}{\left(f_{i_{0}}\right)_{0}}, \frac{\mu_{1}}{f^{\infty}}\right), \lambda f_{i_{0}}<\mu_{1}$ and $\lambda\left(f_{i}\right)^{\infty}<\mu_{1}$ for all $i \in I_{n}$, the result follows from Corollary 2.1.

Corollary 2.2 generalizes Theorem 1.2 with $p=2$ in [7, where $\bar{f}^{\infty}=0,\left(f_{i_{0}}\right)_{0}=$ $\infty$, the norm $|\mathbf{z}|_{1}=\sum_{i=1}^{n}\left|z_{i}\right|$ is used and (1.2) is considered.

As an illustration, we consider the existence of positive solutions of the following system:

$$
\mathbb{L} z_{i}(x)=\left(a_{i} z_{i}^{\alpha_{i}}(x)+b_{i} z_{i}^{\beta_{i}}(x)\right) h_{i}\left(\hat{z}_{i}(x)\right) \quad \text { on } \bar{\Omega}, i \in I_{n},
$$

where $\hat{z}_{i}=\left(z_{1}, \cdots, z_{i-1}, z_{i+1}, \cdots, z_{n}\right)$.

Theorem 2.2. Assume that the following conditions hold:

(i) For each $i \in I_{n}, 0<\alpha_{i}, \beta_{i}<1$ and $a_{i}, b_{i} \geq 0$.

(ii) For each $i \in I_{n}, h_{i}: \mathbb{R}_{+}^{n-1} \rightarrow \mathbb{R}_{+}$is continuous and satisfies

$$
\omega:=\sup \left\{h_{i}\left(\hat{z_{i}}\right): \hat{z}_{i} \in \mathbb{R}_{+}^{n-1} \text { and } i \in I_{n}\right\}<\infty .
$$

(iii) There exists $i_{0} \in I_{n}$ such that $a_{i_{0}}+b_{i_{0}}>0$ and

$$
\xi:=\min \left\{h_{i_{0}}\left(\hat{i_{0}}\right): \hat{i_{0}} \in \mathbb{R}_{+}^{n-1}\right\}>0 .
$$

Then equations (2.19) and (2.2) have a nonzero positive solution in $P$.

Proof. For each $i \in I_{n}$, we define a function $f_{i}: \bar{\Omega} \times \mathbb{R}_{+}^{n} \rightarrow \mathbb{R}_{+}$by

$$
f_{i}(x, \mathbf{z})=\left(a_{i} z_{i}^{\alpha_{i}}+b_{i} z_{i}^{\beta_{i}}\right) h_{i}\left(\hat{z}_{i}\right) .
$$

Let $\varepsilon>0, \sigma=\max \left\{\alpha_{i_{0}}, \beta_{i_{0}}\right\}$ and $0<\rho_{0}<\min \left\{1,\left(\frac{\left(a_{i_{0}}+b_{i_{0}}\right) \xi}{\mu_{1}+\varepsilon}\right)^{\frac{1}{1-\sigma}}\right\}$. Then for $x \in \bar{\Omega}, \mathbf{z} \in \mathbb{R}_{+}^{n}$ with $|\mathbf{z}| \in\left[0, \rho_{0}\right]$ and $z_{i_{0}} \neq 0$,

$$
f_{i_{0}}(x, \mathbf{z}) \geq\left(\frac{a_{i_{0}}}{z_{i_{0}}^{1-\alpha_{i_{0}}}}+\frac{b_{i_{0}}}{z_{i_{0}}^{1-\beta_{i_{0}}}}\right) \xi z_{i_{0}} \geq\left(\frac{a_{i_{0}}}{\rho_{0}^{1-\sigma}}+\frac{b_{i_{0}}}{\rho_{0}^{1-\sigma}}\right) \xi z_{i_{0}} \geq\left(\mu_{1}+\varepsilon\right) z_{i_{0}} .
$$

Hence, $\left(\left(f_{i_{0}}\right)_{0}\right)_{\rho_{0}}$ holds. Since $\overline{f_{i}}(\mathbf{z}) \leq\left[a_{i}|\mathbf{z}|^{\alpha_{i}}+b_{i}|\mathbf{z}|^{\beta_{i}}\right] \omega$ for $i \in I_{n}$, it follows that $\left(f_{i}\right)^{\infty}=\lim \sup _{|\mathbf{z}| \rightarrow \infty} \overline{f_{i}}(\mathbf{z}) /|\mathbf{z}|=0$. The result follows from Theorem 2.1]

As an application of Corollary 2.1, we consider the existence of positive solutions of the following system:

$$
\mathbb{L} z_{i}(x)=a_{i}(x)|\mathbf{z}|^{\alpha_{i}}(x)+b_{i}(x)|\mathbf{z}|(x) \quad \text { on } \bar{\Omega} .
$$

Theorem 2.3. Assume that the following conditions hold:

(i) For each $i \in I_{n}, 0<\alpha_{i}<1$ and $a_{i}, b_{i}: \bar{\Omega} \rightarrow \mathbb{R}_{+}$are continuous.

(ii) There exists $i_{0} \in I_{n}$ such that $\min \left\{a_{i_{0}}(x): x \in \bar{\Omega}\right\}>0$.

(iii) $\left\|b_{i}\right\|<\mu_{1}$ for each $i \in I_{n}$.

Then equations (2.20) and (2.2) have a nonzero positive solution in $P$.

Proof. For each $i \in I_{n}$, we define a function $f_{i}: \bar{\Omega} \times \mathbb{R}_{+}^{n} \rightarrow \mathbb{R}_{+}$by

$$
f_{i}(x, \mathbf{z})=a_{i}(x)|\mathbf{z}|^{\alpha_{i}}+b_{i}(x)|\mathbf{z}| .
$$

Let $\sigma_{1}=\min \left\{a_{i_{0}}(x): x \in \bar{\Omega}\right\}$ and $\sigma_{2}=\min \left\{b_{i_{0}}(x): x \in \bar{\Omega}\right\}$. Then

$$
\underline{f_{i_{0}}}(\mathbf{z}) \geq \sigma_{1}|\mathbf{z}|^{\alpha_{i}}+\sigma_{2}|\mathbf{z}| \quad \text { and }\left(f_{i_{0}}\right)_{0}=\liminf _{|\mathbf{z}| \rightarrow 0^{+}} \underline{f_{i}}(\mathbf{z}) /|\mathbf{z}|=\infty .
$$

Since $\overline{f_{i}}(\mathbf{z}) \leq\left\|a_{i}\right\||\mathbf{z}|^{\alpha_{i}}+\left\|b_{i}\right\||\mathbf{z}|,\left(f_{i}\right)^{\infty}=\lim \sup _{|\mathbf{z}| \rightarrow \infty} \overline{f_{i}}(\mathbf{z}) /|\mathbf{z}| \leq\left\|b_{i}\right\|<\mu_{1}$. The result follows from Corollary 2.1 . 


\section{REFERENCES}

[1] H. Amann, Fixed point equations and nonlinear eigenvalue problems in ordered Banach spaces, SIAM Rev. 18 (1976), 620-709. MR0415432(54:3519)

[2] H. Amann, On the number of solutions of nonlinear equations in ordered Banach spaces, J. Functional Analysis, 11(1972), 346-384. MR0358470 (50:10931)

[3] H. Berestycki and P. L. Lions, Some applications of the method of super and subsolutions, in Bifurcation and Nonlinear Eigenvalue Problems, Lecture Notes in Mathematics, 782, Springer-Verlag, Berlin, 1980, 16-41. MR.572249 (82c:35021)

[4] D. D. Hai, Existence and uniqueness of solutions for quasilinear elliptic systems, Proc. Amer. Math. Soc. 133 (2005), 223-228. MR2085173 (2005g:35073)

[5] D. D. Hai and R. Shivaji, An existence result on positive solutions for a class of semilinear elliptic systems, Proc. Edinb. Math. Soc. Ser. A 134 (2004), 137-141. MR2039906 (2005a:35073)

[6] D. D. Hai and R. Shivaji, An existence result for a class of p-Laplacian systems, Nonlinear Anal. 56 (2004), 1007-1010. MR2038734

[7] D. D. Hai and H. Wang, Nontrivial solutions for p-Laplacian systems, J. Math. Anal. Appl. 330 (2007), 186-194. MR.2302915 (2008a:35068)

[8] P. L. Lions, On the existence of positive solutions of semilinear elliptic equations, SIAM Rev. 24 (1982) (4), 441-467. MR678562 (84a:35093)

[9] R. D. Nussbaum, Eigenvectors of nonlinear positive operators and the linear Krein-Rutman theorem, in Fixed Point Theory (eds. E. Fadell and G. Fournier), Lecture Notes in Math., 886, Springer, 1981, pp. 309-330. MR643014 (83b:47068)

[10] H. Wang, An existence theorem for quasilinear systems, Proc. Edinb. Math. Soc. Ser. A 49 (2006), 505-511. MR2243798 (2008a:35076)

[11] J. R. L. Webb and K. Q. Lan, Eigenvalue criteria for existence of multiple positive solutions of nonlinear boundary value problems of local and nonlocal type, Topol. Methods Nonlinear Anal. 27 (2006) (1), 91-116. MR2236412 (2007f:45006)

Department of Mathematics, Ryerson University, Toronto, Ontario, Canada M5B $2 \mathrm{~K} 3$

E-mail address: klan@ryerson.ca 